\title{
Some Jump Processes in Quantum Field Theory
}

\author{
Roderich Tumulka* and Hans-Otto Georgii ${ }^{\dagger}$
}

\begin{abstract}
A jump process for the positions of interacting quantum particles on a lattice, with time-dependent transition rates governed by the state vector, was first considered by J.S. Bell. We review this process and its continuum variants involving "minimal" jump rates, describing particles as they get created, move, and get annihilated. In particular, we sketch a recent proof of global existence of Bell's process. As an outlook, we suggest how methods of this proof could be applied to similar global existence questions, and underline the particular usefulness of minimal jump rates on manifolds with boundaries.
\end{abstract}

MSC (2000). $\underline{60 \mathrm{~J} 75}, 81 \mathrm{~T} 25$

\section{Introduction}

This contribution deals with Markov jump processes $Q_{t}$ describing the positional time evolution of finitely many interacting quantum particles. These processes are characterized by a specific form of time-dependent jump rates induced by the Schrödinger equation for the quantum state vector $\Psi_{t}$ of the underlying quantum field theory (QFT). As a typical example, suppose that the particles live in the physical three-space $\mathbb{R}^{3}$. The process $Q_{t}$ then takes values in the space of all finite subsets of $\mathbb{R}^{3}$, the configuration space of a variable number of identical particles (corresponding to the Fock space of QFT), and the jumps of $Q_{t}$ describe the creation or annihilation of particles; between these stochastic jumps, $Q_{t}$ evolves deterministically according to some ordinary differential equation governed by $\Psi_{t}$. Alternatively, one may think of quantum particles on a lattice; the jumps of $Q_{t}$ then record all changes of the particle configuration. We will portray several processes of this type, present some common principles, and in particular discuss some results of two recent papers [GT03, DGTZ03c, the work on which was supported by the DFG Priority Program.

As the state vector $\Psi_{t}$ determining the jump rates follows the time-dependent Schrödinger equation, the jump rates themselves are explicitly time-dependent, so that the

*Dipartimento di Fisica and INFN sezione di Genova, Università di Genova, Via Dodecaneso 33, 16146 Genova, Italy. tumulka@mathematik.uni-muenchen.de

${ }^{\dagger}$ Mathematisches Institut, Ludwig-Maximilians-Universität, Theresienstr. 39, 80333 München, Germany. georgii@mathematik.uni-muenchen.de 
processes $Q_{t}$ considered here do not admit an invariant measure. However, the jump rates are designed in such a way that $Q_{t}$ does admit an equivariant measure, namely the quantum distribution $\left|\Psi_{t}\right|^{2}$, which means that $Q_{t}$ has distribution $\left|\Psi_{t}\right|^{2}$ at any time $t$. This is the key feature justifying the particular form of the jump rates, and on the other hand the main fact on which one can build the analysis of these processes. So, the issue here is not the analysis of distributional properties of a given process, but the converse: the equivariant distribution is given, and the objective is to prove the existence of the associated process, and to check that it really does have the equivariant distribution. In GT03, we carried out this program for the case of a discrete configuration space, including in particular a lattice model proposed by J.S. Bell [Bel86]; the main arguments will be sketched in Sect. 4 .

From the probabilistic viewpoint, one has to overcome two difficulties. First, the transition rates exhibit singularities, in that they become ill-defined at certain time and space points. One has to show that the process avoids these singularities. The second (and more important) task is to rule out the possibility of explosion, i.e., the accumulation of infinitely many jumps in finite time. Due to the unbounded growth of the rates near the singularities, the standard methods fail, and one has to use the particular relation between transition rates and equivariant distribution.

Besides the results on the discrete case mentioned above, we will also describe the continuum analogues of Bell's process investigated in DGTZ03a, DGTZ03c; as a special case these include Bohmian mechanics [Boh52, Bel66, BDDGZ95, Dür01] for the appropriate Hamiltonian with a conserved number of particles. On the basis of what we learned from our existence proof for Bell's model, we also propose here some new methods for proving the existence of Bohmian mechanics.

Let us now discuss how the models considered here relate to the topics of other articles in this volume. First, the existence problem for a model of quantum field theory is also the subject of the contribution of S. Albeverio, Y. Kondratiev, M. Röckner, and T. Pasurek. The issue there is the existence, and uniqueness, of Euclidean Gibbs measures for infinitely many interacting quantum spins. These concern an equilibrium setting, and time appears only via path integration to make the connection with the quantum states. The difficulty there is the infinite number of spins, requiring particular assumptions on the interaction. By way of contrast, the models considered here involve only finitely many particles, but in a nonequilibrium situation, and we do not need any particular assumptions on the interaction.

From the methodological side, there is a closer connection to the contributions dealing with population biology, in particular that by R. Höpfner and E. Löcherbach. The similarities concern the creation and annihilation of particles in the vicinity of other particles, and the necessity of proving non-explosion. Also, the space-dependence of the reproduction rates in Höpfner and Löcherbach's article implies non-exponential lifetimes of individuals, just as the time-dependent jump rates considered here imply nonexponential interjump times. However, in our case the paths between the jumps are smooth and deterministic.

This note is organized as follows. In Sect. 2 we derive the fundamental formula 
(8) for minimal jump rates, defining the jump process associated with a certain type of Hamiltonian. This involves consideration of the equivariant probability distribution (4) and probability current (6) provided by quantum theory. In Sect. 3 we explain the connection with Bohmian mechanics and with Bell's model. We also describe the process for a concrete example QFT, introduced in DGTZ03a. In Sect. 4 we sketch the global existence proof for the discrete case, including Bell's model, that we developed in GT03. In Sect. 5 we point out how the methods of GT03 could be adapted to other global existence problems. In Sect. 6 we indicate some perspectives for future research concerning a process for quantum theory on a manifold with boundaries, and the special role the minimal jump rate (8) plays for this process.

\section{Jump Rates Induced by Schrödinger Equations}

We now introduce the class of jump processes we are concerned with, starting with a general framework. For our purposes, a quantum theory is abstractly given by a Hilbert space $\mathscr{H}$ containing the state vectors, a one-parameter group $U_{t}$ of unitary operators on $\mathscr{H}$ defining the time evolution

$$
\Psi_{t}=U_{t} \Psi_{0}
$$

of the state vector, and a measurable space $(\mathcal{Q}, \mathscr{F})$ of configurations describing the locations of particles. $\mathcal{Q}$ is tied to $\mathscr{H}$ via a projection-valued measure (PVM) $P(\mathrm{~d} q)$ on $\mathcal{Q}$ acting on $\mathscr{H}$, i.e., a mapping from the $\sigma$-algebra $\mathscr{F}$ to the family of projection operators on $\mathscr{H}$ that is, like a measure, countably additive (in the sense of the weak operator topology) and normalized, in that $P(\mathcal{Q})=I$, the identity operator on $\mathscr{H}$. If

$\mathscr{H}=L^{2}\left(\mathcal{Q}, \mathbb{C}^{k}\right)$ with respect to some measure on $\mathcal{Q}$ then $\mathscr{H}$ is equipped with a natural PVM, namely $P(B)$ being multiplication by the indicator function of the set $B$. In nonrelativistic quantum mechanics, another way of saying this is that $P$ is the PVM corresponding to the joint spectral decomposition of all position operators.

By Stone's theorem, the unitary operators $U_{t}$ are of the form

$$
U_{t}=\mathrm{e}^{-\mathrm{i} H t / \hbar}
$$

with $H$ a self-adjoint operator on $\mathscr{H}$, called the Hamiltonian. Equations (11) and (2) together correspond to the formal Schrödinger equation

$$
\mathrm{i} \hbar \frac{\mathrm{d} \Psi_{t}}{\mathrm{~d} t}=H \Psi_{t}
$$

We will show how this Schrödinger equation, together with the PVM $P$, gives rise to a natural Markov process on $\mathcal{Q}$.

In this section we focus on the case in which this Markov process is a pure jump process. (Roughly speaking, this will require that the Hamiltonian is an integral operator in the "position representation" defined by $P$; differential operators will be considered in Sect. 3.) So we ask: Is there any distinguished Markovian jump process $\left(Q_{t}\right)$ on $\mathcal{Q}$ 
describing the evolution of the particle configuration, and what are its transition rates? To answer this question we note that the quantum theoretical probability distribution of the configuration at time $t$ is given by

$$
\pi_{t}(\cdot)=\left\langle\Psi_{t} \mid P(\cdot) \Psi_{t}\right\rangle
$$

(We generally assume that $\left\|\Psi_{0}\right\|=1$.) It is therefore natural to stipulate that $\pi_{t}$ is equivariant for $\left(Q_{t}\right)$, meaning that $Q_{t}$ has distribution $\pi_{t}$ at every time $t$. Can one choose some (time-dependent) transition rates $\left(\sigma_{t}\right)$ for $\left(Q_{t}\right)$ to satisfy this requirement of equivariance? Yes indeed, in view of (3) the time derivative of $\pi_{t}$ is given by

$$
\dot{\pi}_{t}(B)=\frac{2}{\hbar} \operatorname{Im}\left\langle\Psi_{t} \mid P(B) H \Psi_{t}\right\rangle=\int J_{t}\left(B, \mathrm{~d} q^{\prime}\right),
$$

where

$$
J_{t}\left(B, B^{\prime}\right)=\frac{2}{\hbar} \operatorname{Im}\left\langle\Psi_{t} \mid P(B) H P\left(B^{\prime}\right) \Psi_{t}\right\rangle
$$

is the quantum theoretical current between two sets $B, B^{\prime} \in \mathscr{F}$. On the other hand, suppose $\left(Q_{t}\right)$ is a pure jump process on $\mathcal{Q}$ jumping at time $t$ with rate $\sigma_{t}\left(B, q^{\prime}\right)$ from $q^{\prime} \in \mathcal{Q}$ to some configuration in $B \in \mathscr{F}$. Then its distribution $\rho_{t}=\mathbb{P} \circ Q_{t}^{-1}$ evolves according to the equation

$$
\dot{\rho}_{t}(B)=\int_{\mathcal{Q}} \sigma_{t}(B, q) \rho_{t}(\mathrm{~d} q)-\int_{B} \sigma_{t}(\mathcal{Q}, q) \rho_{t}(\mathrm{~d} q) .
$$

To satisfy the condition of equivariance we need to find jump rates $\sigma_{t}$ such that the right-hand sides of the evolution equations (5D) and (7) coincide whenever $\rho_{t}=\pi_{t}$. We see that this is the case when $\sigma_{t}$ is given by the Radon-Nikodym derivative

$$
\sigma_{t}\left(\mathrm{~d} q, q^{\prime}\right)=\frac{J_{t}^{+}\left(\mathrm{d} q, \mathrm{~d} q^{\prime}\right)}{\pi_{t}\left(\mathrm{~d} q^{\prime}\right)}=\frac{\left[\frac{2}{\hbar} \operatorname{Im}\left\langle\Psi_{t} \mid P(\mathrm{~d} q) H P\left(\mathrm{~d} q^{\prime}\right) \Psi_{t}\right\rangle\right]^{+}}{\left\langle\Psi_{t} \mid P\left(\mathrm{~d} q^{\prime}\right) \Psi_{t}\right\rangle}
$$

of the positive part $J_{t}^{+}$of $J_{t}$ in its second variable $q^{\prime}$, provided this makes sense. For, the antisymmetry of $J_{t}$ then implies that

$$
\sigma_{t}\left(\mathrm{~d} q, q^{\prime}\right) \pi_{t}\left(\mathrm{~d} q^{\prime}\right)-\sigma_{t}\left(\mathrm{~d} q^{\prime}, q\right) \pi_{t}(\mathrm{~d} q)=J_{t}\left(\mathrm{~d} q, \mathrm{~d} q^{\prime}\right) .
$$

To make formula (8) meaningful one needs some assumptions which roughly require that $H$ is an integral operator in the position representation given by $P$, and $(\mathcal{Q}, \mathscr{F})$ is standard Borel. This is discussed in detail in [DGTZ03C, where (8) was written down for the first time in this generality; special cases had been used before in Bel86, Sud87, DGTZ03a. For the precise conditions we refer to Theorem 1 (Sect. 4.1) and Corollaries 1-3 (Sect. 4.2) of [DGTZ03c]. Under these conditions, formula (8) can (and has to) be read as follows: A priori, $J_{t}$ is a signed bi-measure on $\mathscr{F} \times \mathscr{F}$ (a measure in each of the two variables $q, q^{\prime}$ ). This has to (and then can) be extended to a signed measure on the product $\sigma$-algebra $\mathscr{F} \otimes \mathscr{F}$. The positive part in (8) is then to be understood in the sense of the Hahn-Jordan decomposition of this extended measure. Next one 
notes that, for each $B \in \mathscr{F}, J_{t}(B, \cdot) \ll \pi_{t}$ because $P\left(B^{\prime}\right) \Psi_{t}=0$ whenever $\pi_{t}\left(B^{\prime}\right)=0$. One can thus form the Radon-Nikodym derivatives $\sigma_{t}(B, \cdot)=J_{t}^{+}\left(B, \mathrm{~d} q^{\prime}\right) / \pi_{t}\left(\mathrm{~d} q^{\prime}\right)$, which finally have to be chosen in such a way that $\sigma_{t}$ becomes a measure kernel.

According to our derivation above, the transition rates (8) have been chosen to satisfy the requirement of equivariance. There was, however, still some freedom of choice. The particular rate (8) is singled out by the following additional facts.

1. Suppose there exists a jump process $\left(Q_{t}\right)$ on $\mathcal{Q}$ with rates (8). As is evident from the arguments above, the net probability current of $\left(Q_{t}\right)$ between two sets $B, B^{\prime} \in \mathscr{F}$,

$$
j_{t}\left(B, B^{\prime}\right)=\lim _{\varepsilon \searrow 0} \frac{1}{\varepsilon}\left(\mathbb{P}\left(Q_{t} \in B^{\prime}, Q_{t+\varepsilon} \in B\right)-\mathbb{P}\left(Q_{t} \in B, Q_{t+\varepsilon} \in B^{\prime}\right)\right),
$$

then coincides with the quantum theoretical current $J_{t}$ defined by (6) . Conversely, if $\left(\tilde{Q}_{t}\right)$ is any pure jump process having initial distribution $\pi_{0}$ at time 0 , some jump rates $\tilde{\sigma}_{t}$ and probability current $\tilde{\jmath}_{t}=J_{t}$, it turns out that necessarily $\tilde{\sigma}_{t}\left(\mathrm{~d} q, q^{\prime}\right) \geq$ $\sigma_{t}\left(\mathrm{~d} q, q^{\prime}\right)$ [RS90, BD99, DGTZ03c]. This follows from the minimality of the HahnJordan decomposition. The rates (8) are therefore called the minimal jump rates, and a process with rates (8) is distinguished among all processes with the right probability current by having the least frequent jumps, or the smallest amount of randomness.

2. Always one of the transitions $q^{\prime} \rightarrow q$ or $q \rightarrow q^{\prime}$ is forbidden. More precisely, for every time $t$ there exists a set $S_{t}^{-} \in \mathscr{F} \otimes \mathscr{F}$ which, together with its transposition $S_{t}^{+}$, covers $\mathcal{Q} \times \mathcal{Q}$ (except possibly the diagonal), and such that

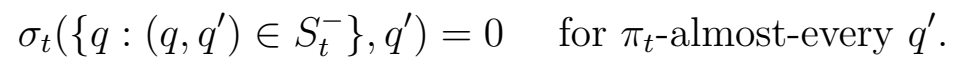

Indeed, by the anti-symmetry of $J_{t}$, its positive and negative part $J_{t}^{+}$and $J_{t}^{-}$ admit supports $S_{t}^{+}$and $S_{t}^{-}$that are transpositions of each other, whence the result follows.

Put more simply, the mechanism is this: When the current $J_{t}\left(\mathrm{~d} q, \mathrm{~d} q^{\prime}\right)$ is positive, meaning that there should be a net flow from $\mathrm{d} q^{\prime}$ to $\mathrm{d} q$, then $\sigma_{t}\left(\mathrm{~d} q, q^{\prime}\right)>0$ and $\sigma_{t}\left(\mathrm{~d} q^{\prime}, q\right)=0$, i.e., only jumps from $q^{\prime}$ to $q$ are allowed; the converse holds in the case of a negative current. Under all rates with $j_{t}=J_{t}$, the minimal rates (8) are characterized by this property.

\section{Bohmian Mechanics and Bell-Type QFT}

In this section we discuss three particular instances in which jump rates of the form (8) play a significant role.

A. Bohmian mechanics as continuum limit of jump processes. Consider nonrelativistic quantum mechanics for $N$ particles: the configuration space is $\mathcal{Q}=\mathbb{R}^{3 N}$, the Hilbert 
space $\mathscr{H}=L^{2}\left(\mathbb{R}^{3 N}, \mathbb{C}^{k}\right)$ and the Hamiltonian

$$
H=-\sum_{i=1}^{N} \frac{\hbar^{2}}{2 m_{i}} \Delta_{i}+V\left(x_{1}, \ldots, x_{N}\right)
$$

with $\Delta_{i}$ the Laplacian acting on the variable $x_{i}, m_{i}$ the mass of the $i$-th particle, and $V$ the potential function (possibly having values in the Hermitian $k \times k$ matrices). We obtain a Markov process on the configuration space in the following way: first discretize space, i.e., replace $\mathbb{R}^{3}$ by a lattice $\Lambda=\varepsilon \mathbb{Z}^{3}$ and the Laplacian $\Delta_{i}$ by the corresponding lattice Laplacian $\Delta_{i}^{\varepsilon}$. We then can consider the jump process $Q_{t}^{\varepsilon}$ on $\Lambda^{N}$ with rates (8). As the lattice shrinks, $\varepsilon \rightarrow 0$, one obtains Sud87, Vin93] in the limit the deterministic process $Q_{t}$ satisfying the ordinary differential equation

$$
\frac{\mathrm{d} Q_{t, i}}{\mathrm{~d} t}=\frac{\hbar}{m_{i}} \operatorname{Im} \frac{\Psi_{t}^{*} \nabla_{i} \Psi_{t}}{\Psi_{t}^{*} \Psi_{t}}\left(Q_{t, 1}, \ldots, Q_{t, N}\right) .
$$

Here $Q_{t, i}$ is the $i$-th component of $Q_{t}$, i.e., the position of the $i$-th particle, $\Psi_{t}$ obeys the Schrödinger equation (3) with Hamiltonian (9), and $\Phi_{1}^{*} \Phi_{2}$ is the inner product in $\mathbb{C}^{k}$. The process (10) is known as Bohmian mechanics [Boh52, Bel66, BDDGZ95, Dür01]. For a suitable other choice of jump rates $V$ in93, also satisfying $j_{t}=J_{t}$ but greater than minimal, one obtains in the continuum limit $\varepsilon \rightarrow 0$ the diffusion process introduced by E. Nelson and known as stochastic mechanics [Nel85].

What makes Bohmian mechanics (or, for that matter, stochastic mechanics) particularly interesting to quantum physicists is that in a Bohmian universe - one in which the particles move according to (10) and the initial configuration is chosen according to the $|\Psi|^{2}$ distribution - the inhabitants find all their observations in agreement with the probabilistic predictions of quantum mechanics - in sharp contrast with the traditional belief that it be impossible to explain the probabilities of quantum mechanics by any theory describing events objectively taking place in the outside world.

B. Bell's jump process for lattice QFT. The study of jump processes with rates (8) has been inspired by Bohmian mechanics, in particular by the wish for a theory similar to Bohmian mechanics covering quantum field theory. The first work in this direction was Bell's seminal paper Bel86]. For simplicity, Bell replaces physical 3-space by a lattice $\Lambda$ and considers a QFT on that lattice. A configuration is specified in his model by the number of fermions $q(x)$ at every lattice site $x$. Thus, with the notation $\mathbb{Z}_{+}=\{0,1,2, \ldots\}$, the configuration space is

$$
\mathcal{Q}=\Gamma(\Lambda):=\left\{q \in \mathbb{Z}_{+}^{\Lambda}: \sum_{x \in \Lambda} q(x)<\infty\right\}
$$

the space of all configurations of a variable (but finite) number of identical particles on the lattice. (In fact, he imposes a bound on the total number of particles and assumes that $\Lambda$ is finite, but this is not necessary.) The PVM $P(\cdot)$ that he suggests arises from the joint spectral decomposition of the fermion number operators $N(x)$ for every lattice site, i.e., $P(q):=P(\{q\})$ is the projection to the joint eigenspace of the (commuting) 
operators $N(x)$ for the eigenvalues $q(x)$. The jump rate Bell uses is the appropriate special case of (8) : the rate of jumping from $q^{\prime}$ to $q$ is

$$
\sigma_{t}\left(q, q^{\prime}\right)=\frac{\left[\frac{2}{\hbar} \operatorname{Im}\left\langle\Psi_{t} \mid P(q) H P\left(q^{\prime}\right) \Psi_{t}\right\rangle\right]^{+}}{\left\langle\Psi_{t} \mid P\left(q^{\prime}\right) \Psi_{t}\right\rangle} .
$$

For studies of Bell's process we refer to Sud87, Vin93, BD99, Col03a, Col03b, DGTZ03c, GT03, and for some numerical simulations and applications to DR03, Den03. We return to it in more detail in the next section.

C. Bohmian mechanics with variable number of particles. A third example of a process for a QFT was considered in DGTZ03a. It arose from an attempt to include particle creation and annihilation into Bohmian mechanics by simply introducing the possibility that world lines of particles can begin and end. That is, the aim is to provide a generalization of the Bohmian motion (10) to a configuration space of a variable number of particles. Here we describe this model in a simplified version. For the numerous similarities between our model process and Bell's discrete process, we called it a "Bell-type QFT." In [DGTZ03c, methods are developed for obtaining a canonical Bell-type process for more or less any regularized QFT.

A configuration of finitely many identical particles can be described by a finite counting measure on $\mathbb{R}^{3}$. Since the coincidence configurations, those in which there are two or more particles at the same location, form a subset of codimension 3, they are basically irrelevant, and it will be convenient to exclude them from the configuration space. What remains, as the space of "simple configurations", is the set of all finite subsets of $\mathbb{R}^{3}$,

$$
\Gamma_{\neq}\left(\mathbb{R}^{3}\right)=\left\{q \subset \mathbb{R}^{3}: \# q<\infty\right\} .
$$

Under the physical conditions prevailing in everyday life, the most frequent type of particle creation and annihilation is the emission and absorption of photons by electrons. This can be described in a model QFT as follows. Particles (photons) move in a Bohmian way and can be emitted and absorbed by another kind of particles (electrons). For simplicity, we will assume here that the electrons remain at fixed locations, given by a finite set $\eta \subset \mathbb{R}^{3}$; the case of moving electrons is described in DGTZ03a. The configuration space is thus the space of photon configurations, $\mathcal{Q}=\Gamma_{\neq}\left(\mathbb{R}^{3}\right)$, and $\Psi_{t}$ a square-integrable complex-valued function on $\mathcal{Q}$; the Hilbert space $\mathscr{H}$ of these functions is known as the bosonic Fock space arising from $L^{2}\left(\mathbb{R}^{3}\right)$.

The Markov process $Q_{t}$ in $\mathcal{Q}$ has piecewise smooth paths. It obeys the deterministic motion (10), interrupted by stochastic jumps. The process is piecewise deterministic in the sense that, conditional on the times of two subsequent jumps and the destination of the first, the path in between these jumps is deterministic. The jumps correspond to creation or annihilation of a photon near some point of $\eta$; in particular, every jump changes the number of photons by one. The process is thus a special kind of a spatial birth-and-death process with moving individuals [Pre76].

The deterministic motion, during which the number of photons is kept constant, is defined by (10); for simplicity, we deviate a little from the physical facts and assume that 
the "photons" have a positive mass $m_{\mathrm{ph}}$. The rate for the transition $q \rightarrow q \cup x:=q \cup\{x\}$, i.e., the creation of a new photon at the location $x \in \mathbb{R}^{3} \backslash q$, has density (with respect to Lebesgue measure $\mathrm{d} x$ )

$$
\sigma_{t}(q \cup x, q)=\frac{\left[\frac{2}{\hbar} \operatorname{Im} \Psi_{t}^{*}(q \cup x)(\# q+1)^{1 / 2} \sum_{y \in \eta} \varphi(x-y) \Psi_{t}(q)\right]^{+}}{\Psi_{t}^{*}(q) \Psi_{t}(q)}
$$

where $\varphi: \mathbb{R}^{3} \rightarrow \mathbb{R}$ is a fixed function, a spherically symmetric, square-integrable potential supported by the ball of radius $\delta>0$. Likewise, for any $x \in q$ the rate for the transition $q \rightarrow q \backslash x:=q \backslash\{x\}$, i.e., annihilation of the photon at $x$, is

$$
\sigma_{t}(q \backslash x, q)=\frac{\left[\frac{2}{\hbar} \operatorname{Im} \Psi_{t}^{*}(q \backslash x)(\# q)^{-1 / 2} \sum_{y \in \eta} \varphi(x-y) \Psi_{t}(q)\right]^{+}}{\Psi_{t}^{*}(q) \Psi_{t}(q)}
$$

These rates, together with vanishing rate for any other transition, are in fact a special case of (8), for a suitable integral operator $H_{I}$ in place of $H$. For the definition of $H_{I}$ and the derivation of the above expressions from (8) we refer to Sect. 3.12 of [DGTZ03c].

Now, $H_{I}$ is not the Hamiltonian of the relevant QFT, but its interaction part; i.e., the complete Hamiltonian is $H=H_{0}+H_{I}$, where $H_{0}$, the free Hamiltonian, is given by

$$
H_{0} \Psi(q)=-\sum_{i=1}^{\# q} \frac{\hbar^{2}}{2 m_{\mathrm{ph}}} \Delta_{i} \Psi(q) .
$$

Observe that there is a correspondence between the splitting $H=H_{0}+H_{I}$ and the two constituents of the process, the motion (10) and the jump rates just given. Deterministic motion corresponds to $H_{0}$ while the jumps correspond to $H_{I}$. Indeed, the minimal process, the one arising as a limiting case from jump processes with minimal rates (8)), associated with $H_{0}$ alone is the continuous motion (10) while the minimal process associated with $H_{I}$ is the pure jump process with the above rates.

This is an instance of the general rule of process additivity: If the minimal process associated with $H_{1}$ has generator $\mathscr{L}_{1, \Psi_{t}}$ and the one associated with $H_{2}$ has generator $\mathscr{L}_{2, \Psi_{t}}$, then the minimal process associated with $H_{1}+H_{2}$ has generator $\mathscr{L}_{1, \Psi_{t}}+\mathscr{L}_{2, \Psi_{t}}$, provided that the (formal) integral kernels of $H_{1}$ and $H_{2}$ have disjoint supports. The sum of the generators of a deterministic flow and of a pure jump process generates the piecewise deterministic process that follows the flow between stochastic jumps. In QFT, it is a typical situation that $H=H_{0}+H_{I}$ where $H_{0}$ is a differential operator associated with continuous motion while $H_{I}$ is an integral operator (often linking different particle numbers) associated with jumps.

\section{Global Existence of Bell's Jump Process}

In this section we deal with Bell's jump process introduced as model $B$ in the last section. As we have shown in GT03, this process exists globally in time. In fact, for 
our proof it is not relevant whether the configuration corresponds to the fermion number operators. We only need that $\mathcal{Q}$ is any countable set, and $P(\cdot)$ a $\mathrm{PVM}$ on $\mathcal{Q}$ acting on $\mathscr{H}$. In fact we can allow that $P(\cdot)$ is a positive-operator-valued measure (POVM), a concept somewhat weaker than a PVM. ${ }^{1}$ Here is our result.

Theorem 1 Let $\mathscr{H}$ be a Hilbert space, $H$ a self-adjoint operator on $\mathscr{H}, \mathcal{Q}$ a countable set, and $P(\cdot)$ a POVM on $\mathcal{Q}$ acting on $\mathscr{H}$. For every initial state vector $\Psi_{0}$ with $\left\|\Psi_{0}\right\|=1$ satisfying

$$
\begin{gathered}
\Psi_{t} \in \operatorname{domain}(H) \quad \forall t \in \mathbb{R}, \\
P(q) \Psi_{t} \in \operatorname{domain}(H) \quad \forall t \in \mathbb{R}, q \in \mathcal{Q}, \\
\int_{t_{1}}^{t_{2}} d t \sum_{q, q^{\prime} \in \mathcal{Q}}\left|\left\langle\Psi_{t} \mid P(q) H P\left(q^{\prime}\right) \Psi_{t}\right\rangle\right|<\infty \quad \forall t_{1}, t_{2} \in \mathbb{R} \text { with } t_{1}<t_{2},
\end{gathered}
$$

there exists a (right-continuous) Markovian pure jump process $\left(Q_{t}\right)_{t \in \mathbb{R}}$ on $\mathcal{Q}$ with transition rates (11) such that, for every $t, Q_{t}$ has distribution $\pi_{t}=\left\langle\Psi_{t} \mid P(\cdot) \Psi_{t}\right\rangle$. This process is unique in distribution.

Some comments are necessary. First of all, how could the process fail to exist globally in time? Two kinds of catastrophes could occur. On the one hand, the jump rate (11) is singular at the nodes of $\Psi$ (i.e., at such $q$ and $t$ for which $\left\langle\Psi_{t} \mid P(q) \Psi_{t}\right\rangle=0$ ). While $Q_{t}$ is sitting on a configuration $q$ it might become a node, and then the process would not know how to proceed. It turns out that this problem does not arise because, with probability one, there is no $t$ at which $Q_{t}$ is a node. This is because the increase of the rates close to the nodes has the positive effect of forcing the process to jump away before the singularity time is reached.

The second kind of possible catastrophe would be an explosion, i.e., an accumulation of infinitely many jumps in finite time. The main task is to show that this does not occur, with probability one. The standard criteria for non-explosion of pure jump processes are confined to transition rates that are homogeneous in time, relying heavily on the fact that the holding times are then exponentially distributed and independent conditionally on the positions; see, e.g., Sect. 2.7 of [Nor97. This conditional independence, however, fails to hold in the case of time-dependent jump rates, and the singularities of Bell's transition rates do not allow any a priori bounds as they were used, e.g., in Pre76, RL53] to exclude explosion. The only thing one knows is that the process is designed to have the prescribed quantum distribution (44) at fixed (deterministic) times, and it is this fact we will exploit. We will sketch our main arguments below.

Steps towards a proof of global existence of Bell's process have also been made by G. Bacciagaluppi [Bac96, BD99]; his approach is, however, very different from ours.

Concerning the technical assumptions (12) on $H, P$, and $\Psi_{0}$ we note the following. For fixed $H$ and $P$, the conditions (12) define a set of "good" initial state vectors $\Psi_{0}$

\footnotetext{
${ }^{1}$ That is, $P$ takes values in the positive (bounded, self-adjoint) operators on $\mathscr{H}$ (instead of the projection operators as a PVM) and shares the countable additivity and normalization of a PVM.
} 
for which we can prove global existence; this set is obviously invariant under the time evolution (11). In fact, when $H$ is a Hilbert-Schmidt operator (i.e., $\operatorname{tr} H^{2}<\infty$ ), the conditions (12) are satisfied for all POVMs $P$ and all $\Psi_{0} \in \mathscr{H}$; this is also true when $H$ is bounded and $\mathcal{Q}$ is finite. (Usually, the Hamiltonian of a lattice QFT is not HilbertSchmidt but can, at least, be approximated by Hilbert-Schmidt operators. And it is not unusual in quantum field theory that Hamiltonians need to be "cut off" in one way or another to make them treatable, or well-defined at all.) Condition (12b) ensures that the expression $P(q) H P\left(q^{\prime}\right) \Psi_{t}$ can be formed, and thus that (11) is well-defined whenever $q^{\prime}$ is not a node.

The main construction is obvious. Starting from any fixed initial time $t_{0}$, the process $Q_{t}$ can be expressed for $t>t_{0}$ in terms of $T_{k}$ and $X_{k}$, the time and the destination of the $k$-th jump after time $t_{0}$, via

$$
Q_{t}=X_{k} \quad \text { if } T_{k} \leq t<T_{k+1}
$$

with $T_{0}:=t_{0}$ and $X_{0}=Q_{t_{0}}$. The variables $T_{k}$ and $X_{k}$ are defined by their conditional distributions:

$$
\begin{aligned}
\mathbb{P}\left(T_{k+1} \in \mathrm{d} t, X_{k+1}=q \mid T_{0}, X_{0}, \ldots, T_{k}, X_{k}\right) & = \\
& \mathbf{1}_{\left\{T_{k}<t\right\}} \sigma_{t}\left(q, X_{k}\right) \exp \left(-\int_{T_{k}}^{t} \sigma_{s}\left(\mathcal{Q}, X_{k}\right) \mathrm{d} s\right) \mathrm{d} t
\end{aligned}
$$

where the role of the "failure rate function" is played by

$$
\sigma_{t}(\mathcal{Q}, q)=\sum_{q^{\prime} \in \mathcal{Q}} \sigma_{t}\left(q^{\prime}, q\right)
$$

the total jump rate, to whatever destination, at $q$.

Here is the reason why the process cannot run into a node. By definition, the conditional probability of remaining at $q$ until at least time $t_{2}$, given that $Q_{t_{1}}=q$, is

$$
\exp \left(-\int_{t_{1}}^{t_{2}} \sigma_{t}(\mathcal{Q}, q) \mathrm{d} t\right)
$$

We want to show that this probability vanishes whenever $q$ is a node at $t_{2}$ but not at any $t$ with $t_{1} \leq t<t_{2}$. Ignoring some technical subtleties, this can be derived by the following simple calculation. Since a sum of positive parts exceeds the positive part of the sum, we conclude from (11) that

$$
\sigma_{t}\left(\mathcal{Q}, q^{\prime}\right)=\sum_{q \in \mathcal{Q}} \frac{\left[\frac{2}{\hbar} \operatorname{Im}\left\langle\Psi_{t} \mid P(q) H P\left(q^{\prime}\right) \Psi_{t}\right\rangle\right]^{+}}{\left\langle\Psi_{t} \mid P\left(q^{\prime}\right) \Psi_{t}\right\rangle} \geq \frac{\left[\frac{2}{\hbar} \operatorname{Im}\left\langle\Psi_{t} \mid H P\left(q^{\prime}\right) \Psi_{t}\right\rangle\right]^{+}}{\left\langle\Psi_{t} \mid P\left(q^{\prime}\right) \Psi_{t}\right\rangle}
$$

Omitting the positive part and using (5) we find

$$
\sigma_{t}\left(\mathcal{Q}, q^{\prime}\right) \geq \frac{-(\mathrm{d} / \mathrm{d} t)\left\langle\Psi_{t} \mid P\left(q^{\prime}\right) \Psi_{t}\right\rangle}{\left\langle\Psi_{t} \mid P\left(q^{\prime}\right) \Psi_{t}\right\rangle}=-\frac{\mathrm{d}}{\mathrm{d} t} \log \left\langle\Psi_{t} \mid P\left(q^{\prime}\right) \Psi_{t}\right\rangle
$$


at every $t$ with $\left\langle\Psi_{t} \mid P\left(q^{\prime}\right) \Psi_{t}\right\rangle>0$. Hence, by the fundamental theorem of calculus,

$$
\int_{t_{1}}^{t_{2}} \sigma_{t}(\mathcal{Q}, q) \mathrm{d} t \geq-\log \left\langle\Psi_{t_{2}} \mid P(q) \Psi_{t_{2}}\right\rangle+\log \left\langle\Psi_{t_{1}} \mid P(q) \Psi_{t_{1}}\right\rangle=\infty
$$

since $q$ is a node at $t_{2}$ (so that the first term is infinite) but not at $t_{1}$ (so that the second term is finite).

Another part of the proof we would like to sketch here is the core of the argument why the jump times cannot accumulate. As a convenient notation, we introduce an additional "cemetery" configuration $\infty$ and set $Q_{t}:=\infty$ for all $t$ after the explosion time $\sup _{k} T_{k}$. Let $S\left(t_{1}, t_{2}\right)$ be the number of jumps that the process performs in the time interval $\left[t_{1}, t_{2}\right]$. The random variable $S\left(t_{1}, t_{2}\right)$ is either a nonnegative integer or infinite. Our assumption (12c) implies in fact that $S\left(t_{1}, t_{2}\right)$ has finite expectation, for all $t_{1}<t_{2}$, and thus is finite almost surely. To see this we use the equation

$$
\mathbb{E} S\left(t_{1}, t_{2}\right)=\int_{t_{1}}^{t_{2}} \sum_{q, q^{\prime} \in \mathcal{Q}} \sigma_{t}\left(q, q^{\prime}\right) \rho_{t}\left(q^{\prime}\right) \mathrm{d} t
$$

with $\rho_{t}\left(q^{\prime}\right)=\mathbb{P}\left(Q_{t}=q^{\prime}\right)$. Intuitively, equation (13) can be understood as follows. $\sigma_{t}\left(q, q^{\prime}\right) \rho_{t}\left(q^{\prime}\right) \mathrm{d} t$ is the probability of a jump from $q^{\prime}$ to $q$ in the infinitesimal time interval $[t, t+\mathrm{d} t]$. Summing over $q$ and $q^{\prime}$, we obtain (the expected total jump rate and thus) the total probability of a jump during $[t, t+\mathrm{d} t]$. Integrating over $t$, we obtain the expected number of jumps. The point of equation (13) is that its right-hand side involves exclusively the one-time quantities $\sigma_{t}$ and $\rho_{t}$. Now, one can deduce from the definition of the process that

$$
\rho_{t}(q) \leq\left\langle\Psi_{t} \mid P(q) \Psi_{t}\right\rangle,
$$

where any case of strict inequality would have to go along with a positive probability $\mathbb{P}\left(Q_{t}=\infty\right)$ of accumulation before $t$. Combining (13) and (14), we obtain

$$
\begin{aligned}
\mathbb{E} S\left(t_{1}, t_{2}\right) & \leq \int_{t_{1}}^{t_{2}} \sum_{q, q^{\prime} \in \mathcal{Q}} \sigma_{t}\left(q, q^{\prime}\right)\left\langle\Psi_{t} \mid P\left(q^{\prime}\right) \Psi_{t}\right\rangle \mathrm{d} t \\
& =\int_{t_{1}}^{t_{2}} \sum_{q, q^{\prime} \in \mathcal{Q}}\left[\frac{2}{\hbar} \operatorname{Im}\left\langle\Psi_{t} \mid P(q) H P\left(q^{\prime}\right) \Psi_{t}\right\rangle\right]^{+} \mathrm{d} t \\
& \leq \frac{2}{\hbar} \int_{t_{1}}^{t_{2}} \sum_{q, q^{\prime} \in \mathcal{Q}}\left|\left\langle\Psi_{t} \mid P(q) H P\left(q^{\prime}\right) \Psi_{t}\right\rangle\right| \mathrm{d} t<\infty
\end{aligned}
$$

by assumption (12c) of Theorem 1. Indeed, this reasoning more or less dictates the assumption (12c).

\section{Other Global Existence Questions}

Variants of the reasoning in the previous paragraph could be used in the future in other global existence proofs. Here is an example concerning the global existence of Bohmian 
mechanics (with fixed number of particles). This was first proved in BDGPZ95] under suitable assumptions on the potential $V$ and the initial wavefunction $\Psi_{0}$. One way in which a solution $Q_{t}$ of (10) may fail to exist globally is by escaping to infinity (i.e., leaving every bounded set in $\mathbb{R}^{3 N}$ ) in finite time. To control this behavior, we suggest to consider an analogue of $S\left(t_{1}, t_{2}\right)$ : Let $D\left(t_{1}, t_{2}\right)$ be the Euclidean distance in $\mathbb{R}^{3 N}$ traveled by $Q_{t}$ between $t_{1}$ and $t_{2}$. Then

$$
\mathbb{E} D\left(t_{1}, t_{2}\right)=\int_{t_{1}}^{t_{2}} \mathrm{~d} t \int_{\mathbb{R}^{3 N}} \mathrm{~d} q\left|v_{t}(q)\right| \rho_{t}(q),
$$

where $v_{t}$ is the Bohmian velocity vector field on $\mathbb{R}^{3 N}$, with $i$-th component given by the right-hand side of (10), and the expectation is taken over the randomness coming from the initial configuration. If this expectation can be shown to be finite, $D\left(t_{1}, t_{2}\right)$ must be almost surely finite. Using $\rho_{t}(q) \leq\left|\Psi_{t}(q)\right|^{2}$, the analogue of (14), we see that the escape to infinity is almost surely excluded provided that

$$
\int_{t_{1}}^{t_{2}} \mathrm{~d} t \int_{\mathbb{R}^{3 N}} \mathrm{~d} q\left|\Psi_{t}^{*}(q) \nabla \Psi_{t}(q)\right|<\infty \quad \text { whenever } t_{1}<t_{2} .
$$

This is a condition analogous to (12c); it is almost equivalent to the assumption A4 of BDGPZ95. The proof there, however, is different, using skillful estimates of the probability flux across suitable surfaces in configuration space-time $\mathbb{R}^{3 N} \times \mathbb{R}$ surrounding the "bad" points (nodes, infinity, points where $\Psi$ is not differentiable). The above argument based on (15) might contribute to a simpler global existence proof.

The global existence question is still open for the Bohm-Dirac law of motion Boh53, BH93, a version of Bohmian mechanics suitable for wavefunctions $\psi$ obeying the Dirac equation. The Dirac equation is a relativistic version of the Schrödinger equation and reads

$$
\mathrm{i} \hbar \frac{\partial \psi}{\partial t}=-\sum_{i=1}^{N}\left(\mathrm{i} c \hbar \alpha_{i} \cdot \nabla_{i} \psi+\beta_{i} m_{i} c^{2} \psi\right)
$$

where $\psi_{t}$ is a function $\mathbb{R}^{3 N} \rightarrow\left(\mathbb{C}^{4}\right)^{\otimes N}, c$ is the speed of light, $m_{i}$ the mass of the $i$ th particle, $\alpha_{i}$ the vector of Dirac alpha matrices acting on the $i$-th spin index of the wavefunction, and $\beta_{i}$ the Dirac beta matrix acting on the $i$-th index. The Bohm-Dirac equation of motion reads

$$
\frac{\mathrm{d} Q_{t, i}}{\mathrm{~d} t}=c \frac{\psi_{t}^{*} \alpha_{i} \psi_{t}}{\psi_{t}^{*} \psi_{t}}\left(Q_{t, 1}, \ldots, Q_{t, N}\right)
$$

where $\phi^{*} \psi$ is the inner product in Dirac spin-space. Since these velocities are bounded by the speed of light, the question of escape to infinity does not arise here. But the question of running into a node does because, like the minimal jump rate (8), the velocity formula (17) is ill-defined at nodes.

This question can be treated in a way analogous to the previous arguments based on (13) and (15). To this end, let $L\left(t_{1}, t_{2}\right)$ be the total variation, between $t_{1}$ and $t_{2}$, of 
$\log \left|\psi_{t}\left(Q_{t}\right)\right|^{2} ;$ in other words,

$$
L\left(t_{1}, t_{2}\right)=\left.\int_{t_{1}}^{t_{2}} \mathrm{~d} t\left|\frac{\mathrm{d}}{\mathrm{d} t} \log \right| \psi_{t}\left(Q_{t}\right)\right|^{2} \mid
$$

It takes values in $[0, \infty]$ and is infinite if the trajectory $Q_{t}$ runs into a node between $t_{1}$ and $t_{2}$. This must be a null event if $L\left(t_{1}, t_{2}\right)$ has finite expectation; for the latter we have the formula

$$
\mathbb{E} L\left(t_{1}, t_{2}\right)=\left.\int_{t_{1}}^{t_{2}} \mathrm{~d} t \int_{\mathbb{R}^{3 N}} \mathrm{~d} q\left|\left(\frac{\partial}{\partial t}+v_{t}(q) \cdot \nabla\right) \log \right| \psi_{t}(q)\right|^{2} \mid \rho_{t}(q),
$$

where $v_{t}$ is the vector field on $\mathbb{R}^{3 N}$ whose $i$-th component is the right-hand side of (17)). Using $\rho_{t}(q) \leq\left|\psi_{t}(q)\right|^{2}$ we obtain

$$
\mathbb{E} L\left(t_{1}, t_{2}\right) \leq\left.\int_{t_{1}}^{t_{2}} \mathrm{~d} t \int_{\mathbb{R}^{3 N}} \mathrm{~d} q\left|\left(\frac{\partial}{\partial t}+v_{t}(q) \cdot \nabla\right)\right| \psi_{t}(q)\right|^{2} \mid .
$$

As the velocities are bounded, the last expression is at most

$$
\int_{t_{1}}^{t_{2}} \mathrm{~d} t \int_{\mathbb{R}^{3 N}} \mathrm{~d} q\left(\left.\left|\frac{\partial}{\partial t}\right| \psi_{t}(q)\right|^{2}|+c| \nabla\left|\psi_{t}(q)\right|^{2} \mid\right) .
$$

Inserting (16) and using that the alpha and beta matrices have norm 1 we see that this in turn is not larger than

$$
\int_{t_{1}}^{t_{2}} \mathrm{~d} t \int_{\mathbb{R}^{3 N}} \mathrm{~d} q\left(2 c\left|\psi_{t}^{*} \nabla \psi_{t}\right|+\frac{2}{\hbar}\left(\sum_{i} m_{i} c^{2}\right) \psi_{t}^{*} \psi_{t}+2 c\left|\psi_{t}^{*} \nabla \psi_{t}\right|\right) .
$$

Since $\left\|\psi_{t}\right\|=1$, the last integral coincides with

$$
\frac{2}{\hbar}\left(\sum_{i} m_{i} c^{2}\right)\left(t_{2}-t_{1}\right)+4 c \int_{t_{1}}^{t_{2}} \mathrm{~d} t \int_{\mathbb{R}^{3 N}} \mathrm{~d} q\left|\psi_{t}^{*} \nabla \psi_{t}\right| .
$$

The question remains under which conditions on $\psi_{0}$ the last term is finite for arbitrary $t_{1}<t_{2}$. This is presumably the case when $\psi_{0}$ lies in Schwartz space (containing all smooth functions $f$ such that $f$ and all its derivatives decay, at infinity, faster than $|q|^{-n}$ for any $\left.n>0\right)$. To work out the proof remains for future research [TT04].

While global existence of the Bohm-Dirac trajectories can presumably be proved with the same methods as used in BDGPZ95 for (10) (estimating the flux across suitable surfaces surrounding the bad points), such a proof requires a lot of effort. It seems that the argument just sketched would be much easier and more elegant.

Another global existence question that is still open is that concerning the process defined in [DGTZ03a] and described above in Part C of Sect. 3, and for similar processes, on a configuration space that is a disjoint union of manifolds, following deterministic trajectories interrupted by stochastic jumps. 


\section{Deterministic Jumps and Boundaries in Configu- ration Space}

In this last section we describe another application of minimal jump rates, one that has not yet been discussed in the literature and that raises some questions for further research. Suppose that the configuration space $\mathcal{Q}$ is a Riemannian manifold with boundaries, or more generally the disjoint union of (at most countably many) Riemannian manifolds with boundaries. We write $\mathcal{Q}=\partial \mathcal{Q} \cup \mathcal{Q}^{\circ}$ where $\partial \mathcal{Q}$ denotes the boundary and $\mathcal{Q}^{\circ}$ the interior.

We develop below an analogue of Bohmian mechanics on $\mathcal{Q}$, consisting of smooth motion interrupted by jumps from the boundary to the interior or vice versa. The jumps from $\partial \mathcal{Q}$ to $\mathcal{Q}^{\circ}$ are deterministic and occur whenever the process hits the boundary. The jumps from $\mathcal{Q}^{\circ}$ to $\partial \mathcal{Q}$ are stochastic, and their rates are fully determined by requiring that (i) the process is Markovian and equivariant, and (ii) the construction is invariant under time reversal, in that the processes associated with $\Psi_{t}$ resp. $\Psi_{-t}^{*}$ are reverse to each other, in distribution. These rates are, in fact, another instance of minimal jump rates.

Configuration spaces with boundaries arise from QFT if a particular kind of "ultraviolet cutoff" is applied, which can be regarded as corresponding to smearing out the charge of an electron over a sphere rather than a ball. Here is an example. Consider again electrons and photons, with the electrons fixed at locations given by the finite set $\eta \subset \mathbb{R}^{3}$, and suppose that photons cannot get closer than a fixed (small) distance $\delta>0$ to an electron, as they get absorbed when they reach that distance. Thus, the available configuration space is

$$
\mathcal{Q}=\left\{q \in \Gamma_{\neq}\left(\mathbb{R}^{3}\right): d(q, \eta) \geq \delta\right\},
$$

where $d(q, \eta)$ is the Euclidean distance of the finite sets $q$ and $\eta$. The space $\mathcal{Q}$ is a countable disjoint union of Riemannian manifolds with boundary,

$$
\mathcal{Q}=\bigcup_{n=0}^{\infty}\{q \in \mathcal{Q}: \# q=n\} .
$$

Its interior is $\mathcal{Q}^{\circ}=\left\{q \in \Gamma_{\neq}\left(\mathbb{R}^{3}\right): d(q, \eta)>\delta\right\}$, and its boundary $\partial \mathcal{Q}=\left\{q \in \Gamma_{\neq}\left(\mathbb{R}^{3}\right)\right.$ : $d(q, \eta)=\delta\}$.

For this or any other configuration space with boundaries, the law of motion

$$
\frac{\mathrm{d} Q_{t}}{\mathrm{~d} t}=v_{t}\left(Q_{t}\right)=\frac{\hbar}{m} \operatorname{Im} \frac{\Psi_{t}^{*} \nabla \Psi_{t}}{\Psi_{t}^{*} \Psi_{t}}\left(Q_{t}\right)
$$

on $\mathcal{Q}^{\circ}$ must be completed by specifying what should happen to the process at the time $\tau$ when it reaches the boundary. (No specification is needed, however, for what should happen when two photons collide, as this has probability zero ever to occur.) The specification we consider here is a deterministic jump law

$$
Q_{\tau+}=f\left(Q_{\tau-}\right)
$$


for a fixed mapping $f: \partial \mathcal{Q} \rightarrow \mathcal{Q}^{\circ}$. In our example (18), the obvious choice of $f$ is

$$
f(q)=\{x \in q: d(x, \eta)>\delta\}
$$

which means that all photons having reached the critical distance $\delta$ to some electron disappear.

Since we want the theory to be reversible, we must also allow for spontaneous jumps from interior points to boundary points. Since we want the process to be an equivariant Markov process, the rate for a jump from $q^{\prime} \in \mathcal{Q}^{\circ}$ to a surface element $\mathrm{d} q \subseteq \partial \mathcal{Q}$ must be, as one can derive,

$$
\sigma_{t}\left(\mathrm{~d} q, q^{\prime}\right)=\frac{\left[n(q) \cdot v_{t}(q)\left|\Psi_{t}(q)\right|^{2}\right]^{+}}{\left|\Psi_{t}\left(q^{\prime}\right)\right|^{2}} \nu\left(\mathrm{d} q, q^{\prime}\right)
$$

where $n(q)$ is the inward unit normal vector to the boundary at $q \in \partial \mathcal{Q}$, the dot $\cdot$ denotes the Riemannian inner product, and $\nu\left(\mathrm{d} q, q^{\prime}\right)$ is the measure-valued function defined in terms of the Riemannian volume measure $\mu$ on $\mathcal{Q}$ and the Riemannian surface area measure $\lambda$ on $\partial \mathcal{Q}$ by

$$
\nu\left(B, q^{\prime}\right)=\frac{\lambda\left(B \cap f^{-1}\left(\mathrm{~d} q^{\prime}\right)\right)}{\mu\left(\mathrm{d} q^{\prime}\right)},
$$

with $B \subseteq \partial \mathcal{Q}$, and the right-hand side denoting a Radon-Nikodym derivative (the existence of which we presuppose). The measure $\nu\left(\cdot, q^{\prime}\right)$ is concentrated on the subset $f^{-1}\left(q^{\prime}\right)$ of the boundary for almost every $q^{\prime}$. For a probability distribution on $\mathcal{Q}$ having a density function $\rho$ with respect to $\mu$, one obtains the following probability transport equation at $q^{\prime} \in \mathcal{Q}^{\circ}$ :

$$
\frac{\partial \rho_{t}}{\partial t}\left(q^{\prime}\right)=-\nabla \cdot\left(\rho_{t} v_{t}\right)\left(q^{\prime}\right)-\sigma_{t}\left(\partial \mathcal{Q}, q^{\prime}\right) \rho_{t}\left(q^{\prime}\right)+\int_{\partial \mathcal{Q}} \nu\left(\mathrm{d} q, q^{\prime}\right)\left[n(q) \cdot v_{t}(q) \rho_{t}(q)\right]^{-}
$$

For equivariance we need that (21), with $\left|\Psi_{t}\right|^{2}$ in place of $\rho_{t}$, has the structure of the transport equation for $\left|\Psi_{t}\right|^{2}$ that follows from the Schrödinger equation (3),

$$
\frac{\partial\left|\Psi_{t}\left(q^{\prime}\right)\right|^{2}}{\partial t}=\frac{2}{\hbar} \operatorname{Im} \Psi_{t}^{*}\left(q^{\prime}\right)\left(H \Psi_{t}\right)\left(q^{\prime}\right)
$$

This is not automatically the case, but it follows from (and therefore suggests) the following boundary condition relating $\left.\Psi_{t}\right|_{\partial \mathcal{Q}}$ to $\left.\Psi_{t}\right|_{\mathcal{Q}^{\circ}}$ : for all $q \in \partial \mathcal{Q}$,

$$
n(q) \cdot \nabla \Psi_{t}(q)=\gamma(q) \Psi_{t}(f(q))
$$

where $\gamma(q)$ is any complex number. ${ }^{2}$ This condition prescribes the normal derivative of the wavefunction on the boundary. Some boundary condition would be needed anyway to define the evolution of the wavefunction, i.e., to select a self-adjoint extension

\footnotetext{
${ }^{2}$ More generally, if $\Psi$ takes values not in $\mathbb{C}$ but in a higherdimensional complex vector space, $\gamma(q)$ would be a $\mathbb{C}$-linear mapping from the value space at $f(q)$ to the value space at $q$.
} 
of the Laplacian; whether (23) actually suffices for this, we have to leave to future research. Note that (23) is a linear condition and thus defines a subspace of the Hilbert space $L^{2}(\mathcal{Q})$. From (23) and (19), one obtains equivariance with respect to the formal Hamiltonian $H=-\frac{\hbar^{2}}{2 m} \Delta+H_{I}$, where

$$
\begin{aligned}
\left\langle\Phi \mid H_{I} \Psi\right\rangle= & \frac{\hbar^{2}}{2 m} \int_{\mathcal{Q}} \mu\left(\mathrm{d} q^{\prime}\right) \int_{\partial \mathcal{Q}} \nu\left(\mathrm{d} q, q^{\prime}\right) \Phi^{*}\left(q^{\prime}\right) \gamma^{*}(q) \Psi(q) \\
& +\frac{\hbar^{2}}{2 m} \int_{\mathcal{Q}} \mu(\mathrm{d} q) \int_{\partial \mathcal{Q}} \nu\left(\mathrm{d} q^{\prime}, q\right) \Phi^{*}\left(q^{\prime}\right) \gamma\left(q^{\prime}\right) \Psi(q) .
\end{aligned}
$$

Furthermore, the jump rate (20) is indeed the minimal jump rate (8) associated with $H_{I}$, thanks to (23).

Let us emphasize the following aspects. First, starting from the picture of a piecewise deterministic process that jumps whenever it hits the boundary, we arrived with remarkable ease at the probability transport equation (21) and thus at the boundary condition (23). Second, we have derived what the interaction Hamiltonian $H_{I}$ is; once the destination mapping $f$ and the corresponding coefficients $\gamma$ had been selected, there was no further freedom. Third, it turned out that the minimal jump rate is the only possible jump rate in this setting. Its very minimality plays a crucial role: a jump to a boundary point $q$ at which the velocity field is pointing towards the boundary, $n(q) \cdot v_{t}(q)<0$, would not allow any continuation of the process since there is no trajectory starting from $q$. The problem is absent if the velocity at $q$ is pointing away from the boundary, $n(q) \cdot v_{t}(q)>0$. (We are leaving out the case $n(q) \cdot v_{t}(q)=0$.) On the other hand, jumps from $q$ to $f(q)$ cannot occur when $v_{t}(q)$ is pointing away from the boundary since in that case there is no trajectory arriving at $q$. Thus, the jumps must be such that at each time $t$, one of the transitions $q \rightarrow f(q)$ or $f(q) \rightarrow q$ is forbidden, and the decision is made by the sign of $n(q) \cdot v_{t}(q)$.

\section{References}

[Bac96] Bacciagaluppi, G.: Topics in the Modal Interpretation of Quantum Mechanics. Ph. D. thesis, University of Cambridge (1996)

[BD99] Bacciagaluppi, G., Dickson, M.: Dynamics for modal interpretations. Found. Phys., 29, 1165-1201 (1999)

[Bel66] Bell, J.S.: On the problem of hidden variables in quantum mechanics. Rev. Mod. Phys., 38, 447-452 (1966). Reprinted in: Bell, J.S.: Speakable and unspeakable in quantum mechanics. Cambridge University Press, Cambridge (1987), p. 1.

[Bel86] Bell, J.S.: Beables for quantum field theory. Phys. Rep., 137, 49-54 (1986). Reprinted in: Bell, J.S.: Speakable and unspeakable in quantum mechanics. Cambridge University Press, Cambridge (1987), p. 173. 
Also reprinted in: Peat, F.D., Hiley, B.J. (eds) Quantum Implications: Essays in Honour of David Bohm. Routledge, London (1987), p. 227. Also reprinted in: Bell, M., Gottfried, K., Veltman, M. (eds) John S. Bell on the Foundations of Quantum Mechanics. World Scientific Publishing (2001), chap. 17.

[BDGPZ95] Berndl, K., Dürr, D., Goldstein, S., Peruzzi, G., Zanghì, N.: On the global existence of Bohmian mechanics. Commun. Math. Phys., 173, 647-673 (1995), and quant-ph/9503013

[BDDGZ95] Berndl, K., Daumer, M., Dürr, D., Goldstein, S., Zanghì, N.: A Survey of Bohmian Mechanics. Il Nuovo Cimento B, 110, 737-750 (1995), and quant-ph/9504010

[Boh52] Bohm, D.: A Suggested Interpretation of the Quantum Theory in Terms of "Hidden" Variables, I and II. Phys. Rev., 85, 166-193 (1952)

[Boh53] Bohm, D.: Comments on an Article of Takabayasi concerning the Formulation of Quantum Mechanics with Classical Pictures. Progr. Theoret. Phys., 9, 273-287 (1953)

[BH93] Bohm, D., Hiley, B.J.: The Undivided Universe: An Ontological Interpretation of Quantum Theory. Routledge, London (1993)

[Col03a] Colin, S.: The continuum limit of the Bell model. quant-ph/0301119

[Col03b] Colin, S.: A deterministic Bell model. Phys. Lett. A, 317, 349-358 (2003), and quant-ph/0310055

[DR03] Dennis, E., Rabitz, H.: Bell trajectories for revealing quantum control mechanisms. Phys. Rev. A, 67, 033401 (2003), and quant-ph/0208109

[Den03] Dennis, E.: Purifying Quantum States: Quantum and Classical Algorithms. Ph.D. thesis, University of California, Santa Barbara (2003)

[Dür01] Dürr, D.: Bohmsche Mechanik als Grundlage der Quantenmechanik. Springer, Berlin (2001)

[DGTZ03a] Dürr, D., Goldstein, S., Tumulka, R., Zanghì, N.: Trajectories and Particle Creation and Annihilation in Quantum Field Theory. J. Phys. A: Math. Gen., 36, 4143-4149 (2003), and quant-ph/0208072

[DGTZ03b] Dürr, D., Goldstein, S., Tumulka, R., Zanghì, N.: Bohmian Mechanics and Quantum Field Theory. quant-ph/0303156

[DGTZ03c] Dürr, D., Goldstein, S., Tumulka, R., Zanghì, N.: Quantum Hamiltonians and Stochastic Jumps. quant-ph/0303056 
[GT03] Georgii, H.-O., Tumulka, R.: Global Existence of Bell's TimeInhomogeneous Jump Process for Lattice Quantum Field Theory. math.PR/0312294 and mp_arc 04-11

[Nel85] Nelson, E.: Quantum Fluctuations. Princeton University Press, Princeton (1985)

[Nor97] Norris, J. R.: Markov chains. Cambridge University Press, Cambridge (1997)

[Pre76] Preston, C. J.: Spatial birth-and-death processes. Bull. Inst. Internat. Statist., 46, no. 2, 371-391, 405-408 (1975)

[RL53] Reuter, G. E. H., Ledermann, W.: On the differential equations for the transition probabilities of Markov processes with enumerably many states. Proc. Cambridge Philos. Soc., 49, 247-262 (1953)

[RS90] Roy, S.M., Singh, V.: Generalized beable quantum field theory. Phys. Lett. B, 234, 117-120 (1990)

[Sud87] Sudbery, A.: Objective interpretations of quantum mechanics and the possibility of a deterministic limit. J. Phys. A: Math. Gen., 20, 1743$1750(1987)$

[TT04] Teufel, S., Tumulka, R.: Global Existence of Bohm-Dirac Trajectories for Nonsingular Potentials. In preparation.

[Vin93] Vink, J.C.: Quantum mechanics in terms of discrete beables. Phys. Rev. A, 48, 1808-1818 (1993) 